\title{
Autoestima en adolescentes implicados en situaciones de acoso escolar
}

\author{
Self-esteem of adolescents involved in bullying situations
}

\author{
Miriam C. Ordóñez-Ordóñez , Marcos R. Narváez \\ Facultad de Psicología, Universidad de Cuenca, Cuenca, Ecuador. \\ Autor para correspondencia: miriam.ordonez@ucuenca.edu.ec \\ Fecha de recepción: 14 de agosto de 2020 - Fecha de aceptación: 7 de septiembre de 2020
}

\section{RESUMEN}

El objetivo de la investigación fue identificar a los adolescentes implicados en situaciones de acoso escolar y el impacto de este fenómeno psicosocial en su autoestima. Se utilizó un muestreo no probabilístico, de tipo no experimental con un enfoque cuantitativo, la muestra estuvo compuesta por 261 estudiantes correspondientes a todos los niveles de bachillerato con edades comprendidas entre los 13 y 18 años de ambos sexos, de una única institución educativa. En la recolección de datos se emplearon las escalas: European Bullying Intervention Project Questionnaire para la identificación de víctimas y agresores y la escala de Autoestima de Rosenberg que valora la autoestima. Se identificó un 31.8\% de adolescentes víctimas, un 5.7\% de agresores y un $9.6 \%$ de agresores victimizados. El sexo masculino se involucraba con mayor frecuencia que el femenino en conductas de victimización; el acoso verbal fue la tipología predominante. En cuanto a la autoestima de los adolescentes, se pudo evidenciar una tendencia al alza (aspectos positivos) siendo los agresores quienes presentaron menores índices de autoestima positiva. En conclusión, se puede evidenciar que la autoestima general de los estudiantes en situaciones de acoso escolar es positiva independientemente de si son víctimas, agresores o espectadores, evidenciándose un nivel alto de satisfacción personal.

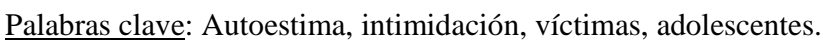

\begin{abstract}
The objective of the research was to identify the adolescents involved in situations of bullying and the impact of this psychosocial phenomenon on their self-esteem. A non-probabilistic sampling was used, as a non-experimental type with a quantitative approach. The sample was composed of 261 students corresponding to all high school levels between the ages of 13 and 18 of both sexes, from a single educational institution. In the data collection the scales of the European Bullying Intervention Project Questionnaire were used for the identification of victims and aggressors and the Rosenberg Self-Esteem scale for the assessment of the self-esteem. The investigation revealed that $31.8 \%$ of the adolescents were victim, $5.7 \%$ aggressors and $9.6 \%$ of victimized aggressors. The male sex was more frequently involved than the female in victimization behaviors and verbal harassment was the predominant typology. As for the adolescents' self-esteem, an upward trend (positive aspects) could be evidenced, with the aggressors who presented lower levels of positive self-esteem. In conclusion, the general self-esteem of students in situations of bullying is positive regardless of whether they are victims, aggressors, or bystanders, evidencing a high level of personal satisfaction.
\end{abstract}

Keywords: Self-esteem, intimidation, victims, adolescents.

\section{INTRODUCCIÓN}

Las agresiones entre pares dentro de las instituciones escolares se conocen como acoso escolar (AE), es una realidad presente en diversos establecimientos de la región latinoamericana, es así como, en Argentina el $61.1 \%$ de los estudiantes se autoidentificaron como víctimas por parte de sus pares (UNICEF, 2010). Fischer (2010) descubrió en instituciones educativas brasileñas, que el $70 \%$ de estudiantes vieron a otros ser acosados en repetidas ocasiones. Las cifras locales en cuanto al $\mathrm{AE}$ evidencian que 6 de cada 10 estudiantes han sido víctimas de acoso escolar (UNICEF, 2008).
El AE es un fenómeno psicosocial que se caracteriza por la intencionalidad de perjudicar o causar daño, por medio del hostigamiento, la opresión y el amedrentamiento repetido hasta el punto de convertirlo en una víctima habitual (Cerezo, 2001). Este acontecimiento no se limita a ser esporádico, puede durar días, meses o incluso años (Olweus, 1997; Ortega, Del Rey, \& Mora, 2001). La importancia de estudiar las consecuencias asociadas al AE es de suma importancia debido a que se puede prevenir, identificar e intervenir en las posibles secuelas asociadas al acoso escolar y su potencial afectación en la autoestima de los adolescentes (Bauzá \& Bennassar, 2009). Las tres características que posibilitan identificar si un estudiante es víctima de AE son: a) cuando es asediado de forma 
repetitiva y sometido durante un tiempo a acciones negativas; b) la presencia de asimetría de poder, en donde a él o a los agresores se les otorga una posición de fuerza y dominio, que puede implicar la fuerza física, económica o simplemente superar en número a la víctima o víctimas (apoyo social); y c) la intencionalidad de causar sufrimiento o dolor, teniendo en consideración que un único hecho aislado no supone que un estudiante se convierta en víctima o en victimario (Olweus, 1995; Cerezo, 2006).

Los agresores o acosadores son estudiantes que tienen conductas agresivas y hostiles, lo utiliza con sus pares para ejercer dominio sobre los demás, para ello hacen uso de comportamientos de amedrentamiento como poner apodos, mofas, insultos o chismes (Estévez, Murgui, \& Musitu, 2009; Griffin \& Gross, 2004). En temas de salud mental los adolescentes que pertenecen a este grupo pueden tener repercusiones durante su vida posterior a la institucional, debido a que son mayormente propensos a futuras conductas delictivas, de manera que la única forma en la que puedan conseguir lo que desean será a través de la violencia (Tobalino, Dolorier, Villa, \& Vargas, 2017). Los estudiantes que se reconocen como víctimas de acoso escolar son aquellos que reciben de manera reiterada cualquier tipo de acoso durante un periodo de tiempo (Delara, 2016), suelen presentar repercusiones en su desempeño escolar, puesto que mantienen un rango de calificaciones entre bajo y medio, además de presentar escasas habilidades sociales y la falta de experiencia para confrontar los comportamientos agresivos (Ortega, 2015). El grupo de los observadores, a quienes Olweus (1995) denomina agresores pasivos, a pesar de no participar en las intimidaciones no toman ninguna iniciativa para contrarrestar el acoso, por lo tanto, se les incluye en una especie de seguidores o secuaces. La clasificación denominada agresor-victimizado hace referencia a la doble función que puede presentar un mismo estudiante, quien, a pesar de autoidentificarse como víctima de $\mathrm{AE}$ también ejerce violencia en contra de sus pares más débiles (Romera, Rey, \& Mora, 2011).

La violencia escolar puede manifestarse de diversas maneras, para Castro (2004), el AE puede adquirir diversas formas, entre ellas: a) verbal, donde destacan los apodos (directo) y hablar mal de alguien (indirecto); b) el maltrato físico a través de amenazas con armas y golpes (directo), pudiéndose evidenciar además el esconder, romper o robar cosas (indirecto); c) la exclusión social, que hace referencia a ignorar a alguien y no dejar que una persona participe en una o varias actividades; y d) el de tipo mixto, que se caracteriza por amenazar a otros con el fin de intimidar, obligar a hacer cosas bajo amenazas (chantaje) y acosar sexualmente. Las repercusiones del AE tienen más fuerza durante la adolescencia debido a que en esta etapa se empieza a formar el autoconcepto, dicha noción hace referencia a las valoraciones que reciben las personas por parte de otros individuos, las cuales presentan mucha significancia personal (Olweus, 1995). Inicialmente los padres juegan un papel fundamental y medular, puesto que son la principal fuente de afecto de los menores, posteriormente lo harán sus maestros y compañeros (Sánchez, Jiménez, \& Merino, 1997), mientras menor sea la edad de la persona, mayor impacto tendrán las agresiones en el autoconcepto de un individuo (Avilés, 2009).

La autoestima ha sido ampliamente estudiada por la relación que tiene con la satisfacción general de un individuo y se resalta como un indicador vital de salud mental (Sánchez et al., 1997; Ulloa, 2003; Polaino, 2003). Este concepto se compone de factores internos (creencias, prácticas o conductas) y de factores externos (verbalizaciones, gestos, experiencias, organizaciones, cultura, padres), que en su conjunto forman la autovaloración de las personas, las cuales limitan o potencializan a un individuo a realizar una actividad y a creer en su potencial (Mézerville, 2005). La autoestima alta se caracteriza por el auto respeto, dichas personas no se sienten inferiores ni superiores a los demás, pueden reconocer sus limitaciones y potencialidades, se muestran prestos a la ayuda y tienen sentimientos de utilidad personal y social, demostrando así una especie de felicidad, madurez y equilibrio (Fanti \& Henrich, 2015). Los adolescentes que se colocan entre una autoestima media o superior tienen mejores herramientas de defensa y confianza para hacerle frente a las agresiones (Salmivalli, Kaukiainen, Kaistaniemi, \& Lagerspetz 1999; Simon, Nail, Swindle, Bihm, \& Joshi, 2017). Rosenberg (1985) expresa que aquellos estudiantes que se encuentren en esta categoría son más susceptibles y manipulables, lo que puede repercutir en que su nivel de autoestima pueda variar hacia el alta o la baja. Aquellos adolescentes que se enmarcan en la autoestima baja se caracterizan por un desconcierto entre lo que razonan, conciben y proyectan hacia el exterior (Salmivalli et al., 1999). El impacto de una baja autoestima se refleja en diversos ámbitos como el rendimiento académico teniendo una mayor probabilidad de padecer síntomas depresivos, ansiedad social o fobia escolar, menor autoconfianza y relaciones conflictivas con sus pares (Hymel \& Swearer, 2015). Otros estudios encontraron características como menor credibilidad en sí mismos, inseguridad para tomar decisiones, aumento en las justificaciones personales, falta de motivación para realizar o alcanzar metas y deterioro de habilidades sociales (Estévez, Martínez, \& Musitu, 2006).

Con el objetivo de identificar los aspectos relacionados al acoso escolar y su posible incidencia en la autoestima, este estudio buscó caracterizar la autoestima de los estudiantes de bachillerato inmersos en situaciones de acoso escolar, además de describir la autoestima de los protagonistas que se incluyen en los diferentes escenarios de AE.

\section{MATERIALES Y MÉTODOS}

Se utilizó un enfoque cuantitativo, de corte trasversal y no experimental debido a que la información se obtuvo de manera estructurada y secuencial en un solo periodo de tiempo con un alcance descriptivo.

Se estudiaron a 261 estudiantes, $46.7 \%$ hombres $(n=46.7)$ y $53.3 \%(n=139)$ mujeres, con edades comprendidas entre los 13 y 18 años, con una edad media de 15.48 años ( $\mathrm{DE}=1.27)$. El $24.9 \%$ de estudiantes fueron de décimo año de educación general básica (EGB), el 23.4\% de primer año de bachillerato general unificado (BGU), el $21.8 \%$ de segundo año (BGU) y el $30.3 \%$ de tercero de bachillerato pertenecientes a una Unidad Educativa rural del cantón Cuenca. Con respecto al tipo de familia se encontró que el $55.2 \%$ de estudiantes pertenecían a una familia nuclear, el $23.8 \%$ a una monoparental materna, el $1.1 \%$ a una monoparental paterno, el $10.7 \%$ a una familia extendida, el $0.8 \%$ a una reconstituida y el $8.4 \%$ a otro tipo de familia. 
Los datos se recolectaron haciendo uso de una ficha sociodemográfica para la caracterización de la población a estudiar, la cual permitió obtener datos como la edad, el sexo, el año de escolaridad y la genealogía familiar. Posteriormente para la identificación de los estudiantes implicados en el acoso escolar se utilizó el instrumento European Bullying Intervention Project Questionnaire (EBIP-Q) en la versión adaptada a la población cuencana por Ordóñez \& Mora (2017), el instrumento consta de 14 ítems, según la frecuencia de cada tipo de agresión; los 7 iniciales corresponde a la identificación de la dimensión de victimización y los 7 posteriores a los de agresión, es de tipo Likert, las puntuaciones van desde 0 a 4 , siendo $0=$ nunca y $4=$ más de una vez a la semana. Las puntuaciones en la consistencia interna (Alpha de Cronbach) reveló 0.76 para los ítems de victimización y 0.75 para los de agresión. Para identificar la autoestima de los estudiantes, se utilizó la Escala de autoestima de Rosenberg en la adaptación realizada por Atienza, Moreno, \& Balaguer (2000), consta de 10 ítems, las 5 primeras tienen afirmaciones positivas y las 5 restantes contienen opiniones negativas, el instrumento tienen un diseño tipo Likert de 1 a 4 , de los cuales 1 hace referencia a estar muy en desacuerdo y 4 a estar muy de acuerdo, con respecto a los puntos de corte: de 30 a 40 corresponde a una autoestima alta, de 25 a 30 una autoestima media y puntuaciones inferiores a 25 hace referencia a una baja autoestima; dicho instrumento presentó una puntuación de 0.77 en el Alfa de Cronbach.

La investigación inició con la aprobación del Comité de Bioética en Investigación del Área de la Salud (COBIAS) de la Universidad de Cuenca, para garantizar la seguridad y confidencialidad de los datos de los participantes. Posteriormente se dedicaron dos días a la sociabilización del proyecto para una adecuada ejecución, para ello se contó con la ayuda del Departamento de Consejería Estudiantil (DECE) quienes permitieron el acercamiento y la vinculación tanto a padres de familia como a los estudiantes confirmando su participación libre y voluntaria mediante la firma de los consentimientos y asentimientos informados, la obtención de los datos se los realizó en una sola jornada educativa. El análisis se realizó en el programa estadístico SPSS 25; se aplicaron pruebas no paramétricas de comparación de medias U-Mann Whitney para dos grupos, la prueba H-Kruskall Wallis para más de dos grupos y el coeficiente de correlación rho de Spearman pues el comportamiento de datos resultó no normal K-S ( $>0.01)$. Además, para establecer la asociación entre el rol de los estudiantes, su curso y sexo se empleó el estadístico Chi-Cuadrado, todas con una significancia del 5\%.

\section{RESULTADOS}

\subsection{Protagonistas del acoso escolar: Implicación, sexo y año de escolaridad}

Se encontró que el $47.1 \%$ de los estudiantes cumplían algún rol de implicación dentro del acoso escolar y el 52.9 $\%$ restante cumplían el papel de espectadores, el 31.8\% resultaron ser víctimas, el $5.7 \%$ agresores y el $9.6 \%$ agresores victimizados; lo que implica que por cada agresor existían aproximadamente 6 víctimas. En cuanto a la variable sexo, el $33.8 \%$ de las mujeres eran víctimas, el $5 \%$ agresoras y el $3.6 \%$ agresoras victimizadas; en tanto que, en el caso de los hombres el $29.5 \%$ resultaron víctimas, el $6.6 \%$ agresores y el $16.4 \%$ agresores victimizados, revelándose una relación significativa de implicación, los hombres se encontraban más vinculados en el acoso escolar $(\mathrm{p}<0.05)$.

En lo referente al nivel académico se evidenció que el 25\% de estudiantes que pertenecían al décimo año de EGB eran víctimas de acoso escolar, en el primer año de bachillerato se encontró un 39.3\% de víctimas (representando la cantidad más alta de víctimas según el curso), en tanto que en el segundo y tercer año de bachillerato se evidenció un $31.6 \%$ de victimización. En el décimo año de EBG se presentó la cantidad más alta de agresores representado el $12.5 \%$ del total de estudiantes de decimo de básica, frente al 2.5\% de tercer año de bachillerato, al $3.5 \%$ de segundo año de bachillerato y al $4.9 \%$ de primer año de bachillerato. Con respecto a los agresores victimizados, el segundo y tercer año de Bachillerato General Unificado (BGU) resultaron ser los niveles educativos con la mayor cantidad de agresores victimizados (12.3\% y $12.7 \%)$, seguido por el primero de bachillerato en el que se presentó en un $8.2 \%$ y finalmente el décimo de EGB con una presencia del $4.7 \%(\mathrm{p}<0.05)$. Detalles en Tabla 1.

\subsection{Tipo de agresión}

Se evidenció que las víctimas recibían principalmente violencia verbal $(\mathrm{M}=4.75 ; \mathrm{DE}=3.27)$, seguido por la violencia física en una intensidad significativamente menor $(\mathrm{M}=1.59$; $\mathrm{DE}=1.64)$. En el caso de los agresores ellos mencionaron, también recibir violencia verbal, siendo esta significativamente menor que las víctimas $(\mathrm{M}=1.37 ; \mathrm{DE}=1.25)$, por su parte los agresores victimizados presentaron altas manifestaciones de violencia verbal $(\mathrm{M}=3.72 ; \mathrm{DE}=3.08)$, mientras que los espectadores presentaron medias inferiores a uno lo que implica una presencia casi nula de violencia. Considerando las expresiones de violencia producida, se encontró, que los agresores afirmaban manifestar principalmente violencia verbal $(\mathrm{M}=2.06 ; \mathrm{DE}=1.46), \mathrm{y}$ física $(\mathrm{M}=1.40 ; \mathrm{DE}=1.18)$, mientras que los agresores victimizados revelaron que principalmente producían violencia física $(\mathrm{M}=2.04 ; \mathrm{DE}=1.46)$, seguido por violencia verbal $(\mathrm{M}=1.96 ; \mathrm{DE}=1.49)$. Finalmente, las víctimas mencionaron producir una violencia casi nula, similar a los espectadores ( $p>0.05)$.

\subsection{Autoestima}

Considerando la autoestima en aspectos positivos y negativos, se encontró que, en cada uno de los protagonistas y espectadores del acoso escolar, los aspectos positivos eran superiores a los aspectos negativos, en general los espectadores eran quienes presentaban mejores niveles de autoestima, seguido por los agresores victimizados, las víctimas y finalmente los agresores. La autoestima positiva en los protagonistas de acoso escolar era superior en las victimas sin representar diferencias significativas con los agresores y agresores victimizados ( $p>0.05$ ), sin embargo, los espectadores presentaron autoestima positiva significativamente superior $(\mathrm{p}<0.05)$. No obstante, la autoestima negativa fue más alta en los agresores $(\mathrm{M}=12.6 ; \mathrm{DE}=3.09)$, seguido por las víctimas y finalmente los agresores victimizados $(\mathrm{M}=11.28 ; \mathrm{DE}=1.74)$; no se encontró una diferencia significativa entre los grupos ( $p>0.05)$. 
Autoestima, manifestaciones de acoso escolar y sexo

Las víctimas mujeres recibían calumnias y chismes en una medida significativamente más alta que los hombres ( $\mathrm{p}<0.01)$; mientras que los agresores victimizados hombres producían golpes significativamente más que las mujeres $(\mathrm{p}<0.05)$. En el caso de los agresores no se encontraron diferencias significativas, en tanto que en los espectadores las mujeres realizaban daños materiales y golpes significativamente más que los hombres, a pesar de que en términos generales en este grupo se evidenciaron manifestaciones muy bajas de recepción y producción de violencia, los detalles en la Tabla 2.
Aspectos positivos y negativos de la autoestima relacionados con las manifestaciones de violencia según el rol en el acoso escolar que sufren las víctimas

En la Tabla 3 se puede visualizar que los aspectos positivos de autoestima del agresor se relacionaron en gran medida y en un sentido inverso con la violencia verbal directa y la violencia verbal indirecta, es decir que, en los agresores a mayores manifestaciones de violencia verbal indirecta y violencia física indirecta, menores eran sus aspectos positivos de autoestima; así también en los espectadores se encontró que entre mayores manifestaciones de actitudes de victimización mixtas de exclusión social y de verbal directa menores eran los aspectos de su autoestima.

Tabla 1. Distribución de la populación des estudiantes según año de escolaridad y rol de implicación.

\begin{tabular}{lcccccccc}
\hline & \multicolumn{2}{c}{$1^{\circ} \mathrm{BGU}$} & \multicolumn{2}{c}{$2^{\circ} \mathrm{BGU}$} & \multicolumn{2}{c}{$3^{\circ} \mathrm{BGU}$} & \multicolumn{2}{c}{$10^{\circ} \mathrm{EGB}$} \\
\cline { 2 - 9 } & $\mathrm{n}$ & $\%$ & $\mathrm{n}$ & $\%$ & $\mathrm{n}$ & $\%$ & $\mathrm{n}$ & $\%$ \\
\hline Victima & 24 & 39.34 & 18 & 31,68 & 25 & 31.63 & 16 & 25.02 \\
Agresor & 3 & 4.93 & 2 & 3.57 & 2 & 2.54 & 8 & 12.53 \\
Agresor victimizado & 5 & 8.22 & 7 & 12.36 & 10 & 12.72 & 3 & 4.74 \\
Espectadores & 29 & 47.57 & 30 & 52.65 & 42 & 53.24 & 37 & 57.82 \\
\hline
\end{tabular}

Nota: BGU=Bachillerato General Unificado; EGB=Educación General Básica

Tabla 2. Manifestaciones de victimización y agresión en los protagonistas de acoso escolar según sexo.

\begin{tabular}{|c|c|c|c|c|c|c|c|c|c|c|c|c|c|c|c|c|c|}
\hline & & \multicolumn{4}{|c|}{ Victimas } & \multicolumn{4}{|c|}{ Agresores } & \multicolumn{4}{|c|}{ Agresores victimizados } & \multicolumn{4}{|c|}{ Espectadores } \\
\hline & & \multicolumn{2}{|c|}{ Mujeres } & \multicolumn{2}{|c|}{ Hombres } & \multicolumn{2}{|c|}{ Mujeres } & \multicolumn{2}{|c|}{ Hombres } & \multicolumn{2}{|c|}{ Mujeres } & \multicolumn{2}{|c|}{ Hombres } & \multicolumn{2}{|c|}{ Mujeres } & \multicolumn{2}{|c|}{ Hombres } \\
\hline & & $\mathrm{M}$ & $\mathrm{DE}$ & $\mathrm{M}$ & $\mathrm{DE}$ & $\mathrm{M}$ & $\mathrm{DE}$ & $\mathrm{M}$ & $\mathrm{DE}$ & $\mathrm{M}$ & $\mathrm{DE}$ & $\mathrm{M}$ & $\mathrm{DE}$ & $\mathrm{M}$ & $\mathrm{DE}$ & $\mathrm{M}$ & $\mathrm{DE}$ \\
\hline \multirow{7}{*}{ 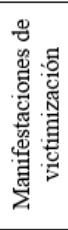 } & Golpes & 0.7 & 1 & 0.7 & 0.9 & 0 & 0 & 0 & 0 & 1 & 1 & 0.9 & 1.2 & 0.1 & 0.3 & 0.1 & 0.3 \\
\hline & Insultos & 1.7 & 1.4 & 1.6 & 1.4 & 0.4 & 0.5 & 0.1 & 0.4 & 1.2 & 1.3 & 1.5 & 1.4 & 0.2 & 0.4 & 0.3 & 0.5 \\
\hline & Calumnia & $1.8^{* *}$ & 1.4 & 0.9 & 1.2 & 0.4 & 0.5 & 0.3 & 0.5 & 1 & 1.4 & 0.7 & 0.9 & 0.3 & 0.4 & 0.2 & 0.4 \\
\hline & Amenazas & 0.5 & 1 & 0.4 & 0.9 & 0.1 & 0.4 & 0.1 & 0.4 & 0.6 & 1.3 & 0.7 & 1.2 & 0.0 & 0.2 & 0.1 & 0.4 \\
\hline & $\begin{array}{l}\text { Robos, daños } \\
\text { materiales }\end{array}$ & 1 & 1.1 & 0.8 & 1 & 0.3 & 0.5 & 0 & 0 & 1.2 & 1.3 & 0.7 & 1 & $0.1^{*}$ & 0.3 & 0.3 & 0.4 \\
\hline & Dejar de lado & 0.9 & 1.4 & 0.8 & 1 & 0.3 & 0.5 & 0 & 0 & 0.6 & 0.9 & 1 & 1.3 & 0.2 & 0.4 & 0.2 & 0.4 \\
\hline & Chismes & $2.1^{* *}$ & 1.4 & 1 & 1.2 & 0.6 & 0.5 & 0.5 & 0.5 & 1.6 & 0.9 & 1.5 & 1.5 & 0.3 & 0.5 & 0.3 & 0.5 \\
\hline \multirow{7}{*}{ 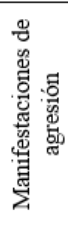 } & Golpes & 0.1 & 0.3 & 0.2 & 0.4 & 0.7 & 1 & 1.1 & 1 & 0.4 & 0.9 & $2.1^{*}$ & 1.4 & $0.1 * *$ & 0.2 & 0.2 & 0.5 \\
\hline & Insultos & 0 & 0.2 & $0.2^{*}$ & 0.4 & 0.6 & 0.8 & 1.1 & 0.8 & 0.6 & 0.5 & 1.1 & 1 & 0 & 0.2 & 0.1 & 0.3 \\
\hline & Calumnia & 0 & 0.2 & 0 & 0 & 0.9 & 1.2 & 0.5 & 0.8 & 0.4 & 0.9 & 0.5 & 0.8 & 0.1 & 0.2 & 0.1 & 0.3 \\
\hline & Amenazas & 0 & 0.2 & 0 & 0.2 & 0.3 & 0.8 & 0.8 & 0.9 & 0 & 0 & 0.5 & 0.9 & 0 & 0.1 & 0.1 & 0.2 \\
\hline & $\begin{array}{l}\text { Robos, daños } \\
\text { materiales }\end{array}$ & 0 & 0 & $0.1^{* *}$ & 0.4 & 0.4 & 0.8 & 0.5 & 0.8 & 0.4 & 0.9 & 0.3 & 0.6 & 0 & 0.2 & 0 & 0.2 \\
\hline & Dejar de lado & 0.1 & 0.3 & 0.1 & 0.4 & 1 & 1.7 & 0.6 & 1.1 & 1.4 & 1.3 & 0.5 & 0.8 & 0.2 & 0.4 & 0.2 & 0.4 \\
\hline & Chismes & 0 & 0.2 & 0.1 & 0.3 & 0.1 & 0.4 & 0.8 & 1 & 0.6 & 0.5 & 0.5 & 0.8 & 0 & 0.2 & 0.1 & 0.3 \\
\hline
\end{tabular}

Tabla 3. Relación entre actitudes de victimización y aspectos positivos y negativos de autoestima según protagonista de acoso.

\begin{tabular}{|c|c|c|c|c|c|c|c|c|}
\hline \multirow[b]{2}{*}{ Aspecto } & \multirow[b]{2}{*}{ Protagonista } & FD & VD & VI & $\mathrm{M}$ & FI & Ex & VD \\
\hline & & Golpes & Insultos & Calumnia & Amenazas & $\begin{array}{l}\text { Robos, } \\
\text { daños } \\
\text { materiales }\end{array}$ & $\begin{array}{l}\text { Dejar de } \\
\text { lado }\end{array}$ & Chismes \\
\hline \multirow{4}{*}{ 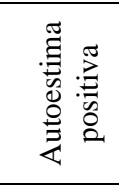 } & Víctima & -0.16 & -0.06 & 0.00 & 0.13 & 0.05 & -0.05 & 0.04 \\
\hline & Agresor & - & $-0.52 *$ & -0.32 & -0.45 & $-0.68 * *$ & -0.45 & -0.15 \\
\hline & $\begin{array}{l}\text { Agresor } \\
\text { victimizado }\end{array}$ & -0.14 & 0.20 & -0.12 & -0.03 & -0.01 & -0.11 & -0.07 \\
\hline & Espectadores & -0.06 & -0.15 & -0.03 & $-0.25 * *$ & $-0.18 *$ & $-0.18^{*}$ & $-0.17 *$ \\
\hline \multirow{4}{*}{ 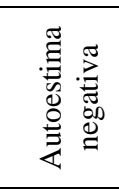 } & Víctima & 0.19 & 0.08 & 0.17 & 0.17 & 0.15 & $0.31 * *$ & 0.12 \\
\hline & Agresor & - & 0.08 & 0.28 & -0.01 & 0.11 & -0.07 & $0.54 *$ \\
\hline & $\begin{array}{l}\text { Agresor } \\
\text { victimizado }\end{array}$ & 0.14 & 0.08 & 0.29 & 0.26 & 0.15 & 0.07 & 0.04 \\
\hline & Espectadores & -0.07 & -0.08 & -0.07 & 0.10 & -0.09 & 0.00 & 0.06 \\
\hline
\end{tabular}

Nota: FD=Fisíca directa, VD=Verbal directa, VI=Verbal indirecta, M=Meido, FI=Fisíca indirecta, Ex=Excluir, VD=Verbal directa, $* \mathrm{p}<0.05, * * \mathrm{p}<0.01$. 
Tabla 4. Relación entre actitudes de victimización y aspectos positivos y negativos de autoestima según protagonista de acoso.

\begin{tabular}{|c|c|c|c|c|c|c|c|c|}
\hline \multirow[b]{2}{*}{ Aspecto } & \multirow[b]{2}{*}{ Protagonista } & FD & VD & VI & $\mathrm{M}$ & FI & Ex & VD \\
\hline & & Golpes & Insultos & Calumnia & Amenazas & $\begin{array}{l}\text { Robos, } \\
\text { daños } \\
\text { materiales }\end{array}$ & $\begin{array}{l}\text { Dejar de } \\
\text { lado }\end{array}$ & Chismes \\
\hline \multirow{4}{*}{ 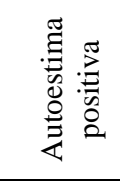 } & Víctima & 0.006 & -0.077 & 0.077 & 0.130 & -0.059 & -0.006 & 0.011 \\
\hline & Agresor & 0.167 & 0.513 & -0.411 & -0.032 & -0.144 & -0.195 & 0.449 \\
\hline & $\begin{array}{l}\text { Agresor } \\
\text { victimizado }\end{array}$ & 0,070 & $0.406^{*}$ & 0.149 & 0.016 & 0.029 & -0.372 & -0.138 \\
\hline & Espectadores & $-0.26^{* *}$ & $-0.212^{*}$ & -0.101 & -0.059 & $-0.203^{*}$ & -0.066 & 0.014 \\
\hline \multirow{4}{*}{ 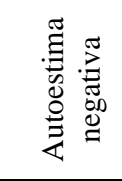 } & Víctima & 0.062 & 0.034 & 0.086 & 0.041 & 0.002 & -0.025 & 0.053 \\
\hline & Agresor & -0.226 & 0.006 & 0.118 & 0.144 & 0.243 & 0.199 & 0.494 \\
\hline & $\begin{array}{l}\text { Agresor } \\
\text { victimizado }\end{array}$ & 0.176 & $-0.474^{*}$ & -0.061 & 0.220 & 0.040 & 0.078 & 0.179 \\
\hline & Espectadores & 0.052 & -0.063 & 0.001 & -0.014 & 0.128 & 0.038 & 0.006 \\
\hline
\end{tabular}

Nota: FD=Fisíca directa, VD=Verbal directa, VI=Verbal indirecta, $\mathrm{M}=$ Meido, FI=Fisíca indirecta, Ex=Excluir, VD=Verbal directa, $* \mathrm{p}<0.05, * * \mathrm{p}<0.01$.

Se observó además que los aspectos negativos de autoestima de las víctimas se encontraban positivamente relacionadas con las manifestaciones de victimización ligadas a la exclusión social, es decir, que ha mayores manifestaciones de exclusión social percibida por los participantes, mayores eran los aspectos negativos del autoestima de los estudiantes, finalmente en los agresores se encontró también una relación importante positiva con la violencia directa (manifestación de victimización), por tanto, a mayor violencia verbal directa, mayores aspectos negativos de autoestima.

En lo referente a las actitudes de agresión se observó que en los agresores victimizados las actitudes de violencia verbal directa se encontraban directamente relacionadas con los aspectos positivos de autoestima de los agresores victimizados, es decir, que a mayor violencia directa, mayores manifestaciones de autoestima positiva, mientras que en los espectadores la violencia física directa e indirecta y la verbal directa (insultos) se encontraban negativamente relacionadas con los aspectos de autoestima positivo. En cuanto a las manifestaciones de violencia verbal directa producidas (manifestaciones de agresión), estas se vincularon fuertemente y en un sentido inverso con los aspectos negativos de la autoestima de los agresores victimizados (ver Tabla 4).

\section{DISCUSIÓN}

Los datos que se encontraron en la investigación exhiben las diversas características de los protagonistas dentro del Acoso Escolar (AE) y la función de la autoestima dentro de este fenómeno psicosocial, para ello, el primer objetivo pretendió identificar a los estudiantes que protagonizan situaciones de $\mathrm{AE}$ y los diversos roles que se suscitan dentro de una institución educativa, de esta manera, se obtuvo que casi el $50 \%$ de todos los estudiantes encuestados ejercían o recibían uno o más tipos de violencia, implicación que en el estudio de Quintana, Montgomery, \& Malaver (2014), son explicados basándose en que las tasas elevadas de acoso escolar se deben a una falta de adaptación y una incongruencia entre los conocimientos que adquieren previamente los adolescentes en sus hogares y contexto social con los que se enfrentan en las situaciones cotidianas dentro de los espacios educativos. Obtener por tanto puntuaciones elevadas en los índices de AE podría deberse a una escasa gama de estrategias de afrontamiento que permita a los adolescentes efectuar una adecuada respuesta ante una situación de agresión (Ortega, 2015).

Se evidenció que el mayor número de estudiantes que ejercían y recibían algún tipo de $\mathrm{AE}$ pertenecían al primero de bachillerato y comprendían un rango de edad entre los 14 y 15 años, una variable interviniente podría ser en este caso en particular la generación y reorganización de los estudiantes con nuevos compañeros de aula, puesto que en la Unidad Educativa, cuando los estudiantes llegan a este nivel educativo se establece una reorganización de los cursos de manera aleatoria, para de esta manera fomentar una integración con los demás estudiantes, es así, como se establecen nuevos cursos integrados por nuevos compañeros. Estos datos resultan similares al estudio de Herrera, Romera, \& Ortega (2017), autores que observaron que los mayores índices de $\mathrm{AE}$ se obtuvieron en los alumnos de 15 años. En cuanto al curso, los datos evidencian que la intensidad y la frecuencia de AE disminuyen en los cursos superiores, esto concuerda con otros estudios realizados que develaron datos similares, pues, los cursos inferiores presentan mayores índices de $\mathrm{AE}$, expresando que a menor edad los estudiantes experimentan mayores problemas relacionales (Cassiani, Gómez, Cubides, \& Hernández, 2014; Ordóñez, Mora, \& Shephard, 2016). En cuanto al sexo, se visualizó que los estudiantes de sexo masculino se encontraban mayormente vinculados en situaciones de acoso escolar, data que coincide con el estudio de Romera, García, \& Ortega (2014) el cual devela una mayor implicación del sexo masculino explicándolo en función a las gratificaciones positivas que reciben por parte de sus pares, pues, lejos de ser rechazados sus comportamientos reciben elogios.

El segundo objetivo pretendió analizar los aspectos positivos y negativos de la autoestima en los estudiantes inmersos en situaciones de acoso escolar, frente a lo cual se evidenció que los adolescentes presentan una mayor tendencia hacia los aspectos positivos de la autoestima. O’Moore \& Kirkham (2001) explican que encontrarse implicados en las diversas posturas del AE, no es un predictor de una baja autoestima, puesto que, no es la 
única esfera en la vida de una persona, si bien el ámbito académico es uno de los principales, los dominios familiares, culturales, educativos y el propio autoconcepto evitarían una afectación en la autoestima global de un individuo. En lo referente a la relación entre acoso escolar y la autoestima de los estudiantes protagonistas de acoso escolar, se encontró que el tipo de violencia más frecuente es de tipo verbal, resultando ser mayoritariamente los agresores quienes son más susceptibles a una baja autoestima. Una investigación realizada por Gondolf (1987) expresó que los estudiantes que se identifican como agresores tienen una tendencia hacia la autoestima baja, puesto que para ellos el autoconcepto se afianza en la opinión de sus pares y la gratificación que conlleva obtener una posición de dominancia sobre otro individuo, sin embargo, de manera opuesta al no sentirse respaldados su autoestima puede deteriorarse significativamente. Se evidenció además que los estudiantes que se identificaron como víctimas obtuvieron menores puntuaciones en su autoestima, Ortega (2015), considera que este fenómeno se suscita por el rechazo y presión social que ejercen tanto los agresores como los espectadores, normalizando el AE y excluyendo a aquellos que no pertenecen a su círculo de amistad más cercano.

Los hallazgos de esta investigación presentan significativa importancia en el ámbito educativo y social, dado que pueden servir como línea base de procesos investigativos, preventivos $o$ de intervención psicoeducativa $y$ psicosocial frente a situaciones de AE.

\section{CONCLUSION}

En términos generales se pudo evidenciar que casi la mitad de los estudiantes cumplían algún rol dentro del $\mathrm{AE}$, siendo los varones quienes presentaron una mayor implicación en las dinámicas de AE. Los cursos que presentaron mayores índices de agresiones fueron aquellos que pertenecían a los décimos cursos de EGB. En cuanto al tipo de acoso que mayormente se presentó fue el de tipo verbal, pues estuvo presente en víctimas, agresores y agresores victimizados, en segundo lugar, se encontró el físico. En lo referente a la victimización, las mujeres recibían mayores agresiones de tipo verbal indirecto, por medio de chismes, en cuanto a los hombres el tipo de acoso más frecuente fue el de tipo verbal directo por medio del insulto. En torno a la variable de autoestima en los alumnos, los aspectos positivos primaron en la mayoría de los participantes siendo los espectadores poseedores de aspectos positivos en su autovaloración, seguido por las víctimas y finalmente los agresores, aunque sin mayores diferencias entre los diversos grupos etarios. Respecto a la relación del AE y la autoestima, los agresores recibían mayores insultos y robos lo cual impacto eventualmente reduciendo su autoestima, en las victimas la variable que mayormente influyo en la disminución de su autoestima fue la exclusión, seguida por los chismes.

Las limitaciones de este estudio se direccionan al uso y autoaplicación de las encuestas, además existen escasos estudios que permitan una adecuada contrastación de los resultados obtenidos con poblaciones de características sociodemográficas similares. Las fortalezas se las obtuvo al establecer una sólida base investigativa con el objetivo de realizar otros estudios que incluya poblaciones institucionalizadas en circunstancias similares.

\section{AGRADECIMIENTO}

Este estudio se realizó en el marco del proyecto «Estrategias de afrontamiento de los niños víctimas frente a situaciones de acoso escolar y cyberbullying», ganador del XV concurso de proyectos de investigación del Departamento de Investigación de la Universidad de Cuenca (DIUC) a quien dirigimos nuestro agradecimiento. Un agradecimiento también muy especial a la Coordinación Zonal 6 de Educación y a las autoridades educativas y estudiantes de la Institución Educativa participante, quienes posibilitaron la ejecución de la presente investigación.

\section{REFERENCIAS}

Atienza, F., Moreno, Y., \& Balaguer, I. (2000). Análisis de la dimensionalidad de la Escala de Autoestima de Rosenberg en una muestra de adolescentes valencianos. Revista Psicología Universitas Tarraconensis, 22, 29-42.

Avilés, J. (2009). Victimización percibida y bullying. Factores diferenciales entre víctima. Boletín de Psicología, 95, 7-28.

Bauzá, J., \& Bennassar, M. del C. F. (2009). Adolescencia y desarrollo emocional en la sociedad actual. International Journal of Developmental and Educational Psychology, 1(1), 235-242.

Cassiani, C., Gómez, J., Cubides, A., \& Hernández, M. (2014). Prevalencia de bullying y factores relacionados en estudiantes de bachillerato de una institución educativa de Cali, Colombia. Revista de Salud Pública, 16(1), 14-26.

https://doi.org/10.15446/rsap.v16n1.43490

Castro, A. (2004). Desaprender la violencia: Un nuevo desafío académico (1a ed.). Buenos Aires, Argentina: Bonum.

Cerezo, F. (2001). Variables de personalidad asociadas en la dinámica bullying (agresores versus víctimas) en niños y niñas de 10 a 15 años. Anales de Psicología, 17(1), 37-43.

Cerezo, F. (2006). Violencia y victimización entre escolares. El bullying: Estrategias de identificación y elementos para la intervención a través del Test Bull-S. Electronic Journal of Research in Education Psychology, 4(4), 333-352. https://doi.org/10.25115/ejrep.v4i9.1192

DeLara, E. (2016). Bullying scars: The impact on adult life and relationships. Oxford, UK: Oxford University Press.

Estévez, E., Martínez, B., \& Musitu, G. (2006). La autoestima en adolescentes agresores y víctimas en la escuela: La perspectiva multidimensional. Intervención Psicosocial, 15(2), 223-232. https://doi.org/10.4321/S1132-05592006000200007 
Estévez, E., Murgui, S., \& Musitu, G. (2009). Psychological adjustment in bullies and victims of school violence. European Journal of Psychology of Education, 24(4), 473-483. https://doi.org/10.1007/BF03178762

Fanti, K., \& Henrich, C. (2015). Effects of self-esteem and narcissism on bullying and victimization during early adolescence. The Journal of Early Adolescence, 35(1), 5-29. https://doi.org/10.1177/0272431613519498

Fischer, R. (2010). Bullying escolar no brasil. Disponible en https://docplayer.com.br/259085Pesquisa-bullying-escolar-no-brasil-relatorio-final.html

Gondolf, E. W. (1987). Evaluating programs for men who batter: Problems and prospects. Journal of Family Violence, 2(1), 95-108. https://doi.org/10.1007/BF00976373

Griffin, R. S., \& Gross, A. M. (2004). Childhood bullying: Current empirical findings and future directions for research. Aggression and Violent Behavior, 9(4), 379-400. https://doi.org/10.1016/S1359-1789(03)00033-8

Herrera, M., Romera, E., \& Ortega, R. (2017). Bullying y cyberbullying en Colombia; coocurrencia en adolescentes escolarizados. Revista Latinoamericana de Psicología, 49(3), 163-172. https://doi.org/10.1016/j.rlp.2016.08.001

Hymel, S., \& Swearer, S. M. (2015). Four decades of research on school bullying: An introduction. American Psychologist, 70(4), 293-299. https://doi.org/10.1037/a0038928

Mézerville, G. (2005). Ejes de salud mental: Los procesos de autoestima, dar y recibir afecto y adaptación al estrés. México: Trillas.

O’Moore, M., \& Kirkham, C. (2001). Self-esteem and its relationship to bullying behaviour. Aggressive Behavior, 27(4), 269-283. https://doi.org/10.1002/ab.1010

Olweus, D. (1995). Bullying or peer abuse at school: Facts and intervention. Current Directions in Psychological Science, 4(6), 196-200. https://doi.org/10.1111/1467-8721.ep10772640

Olweus, D. (1997). Bully and victim problems in school: Facts and intervention. European Journal of Psychology of Education, 12(4), 495-510. https://doi.org/10.1007/BF03172807

Ordóñez, M., \& Mora, C. (2017). Adaptación del European Bullying Intervention Project Questionnaire (EBIPQ) al Contexto Cuencano.

Ordóñez, M., Mora, C., \& Shephard, B. (2016). Estudio transversal: Variable asociadas al acoso escolar. Revista Médica del Hospital José Carrasco Arteaga, 8(1), 44-52. https://doi.org/10.14410/2016.8.1.ao.08

Ortega, R. (2015). Convivencia y ciber-convivencia, un modelo educativo para la prevención del acoso y ciberacoso escolar. Madrid, España: Ed. Antonio Machado Libros.

Ortega, R., Del Rey, R., \& Mora, J. (2001). Violencia entre escolares conceptos y etiquetas verbales que definen el fenómeno del maltrato entre iguales. Revista Interuniversitaria de Formación del Profesorado, 41, 95-113.
Polaino, A. (2003). Familia y autoestima. Disponible en https://educaenvirtudes.files.wordpress.com/ 2010/10/familia_autoestima.pdf

uintana, A., Montgomery, W., \& Malaver, C. (2014). Modos de afrontamiento y conducta resiliente en adolescentes espectadores de violencia entre pares. Revista de Investigación en Psicología, 12(1), 153-171. https://doi.org/10.15381/rinvp.v12i1.3788

Romera, E., García, C., \& Ortega, R. (2014). Acoso en educación primaria: Factores de personalidad y de contexto entre iguales. Revista INFAD de Psicología. International Journal of Developmental and Educational Psychology, 2(1), 383-384. https://doi.org/10.17060/ijodaep.2014.n1.v2.453

Romera, E. M., Rey, R. D., \& Ortega, R. (2011). Factores Asociados a la Implicación en Bullying: Un Estudio en Nicaragua. Psychosocial Intervention, 20(2), 161-170. https://doi.org/10.5093/in2011v20n2a4

Rosenberg, M. (1985). Comunicación no Violenta. Buenos Aires, Argentina: Gran Aldea Editores.

Salmivalli, C., Kaukiainen, A., Kaistaniemi, L., \& Lagerspetz, K. M. J. (1999). Self-Evaluated SelfEsteem, Peer-Evaluated Self-Esteem, and Defensive Egotism as Predictors of Adolescents' Participation in Bullying Situations. Personality and Social Psychology Bulletin, 25(10), 1268-1278. https://doi.org/10.1177/0146167299258008

Sánchez, G., Jiménez, F., \& Merino, V. (1997). Autoestima y del autoconcepto en adolescentes: Una reflexión para la orientación educativa. Revista de Psicología, XV(2), 21 págs. Disponible en http://revistas.pucp.edu.pe/index.php/psicologia/article/ view/5958

Simon, J. B., Nail, P. R., Swindle, T., Bihm, E. M., \& Joshi, K. (2017). Defensive egotism and self-esteem: A cross-cultural examination of the dynamics of bullying in middle school. Self and Identity, 16(3), 270-297. https://doi.org/10.1080/15298868.2016.1232660

Tobalino, D., Dolorier, R., Villa, R., \& Vargas, I. (2017). Acoso escolar y autoestima en estudiantes de educación primaria de Perú. Revista de Ciencias Humanes y Sociales, 33(84), 359-377.

Ulloa, N. (2003). Niveles de autoestima en adolescentes institucionalizados. Tesis de Licenciado en Enfermería, 70 págs. Facultad de Medicina, Escuela de Enfermería, Universidad Austral de Chile. Disponible en http://cybertesis.uach.cl/tesis/uach/2003/fmu.42n/doc/f mu.42n.pdf

UNICEF. (2008). Estudio del Acoso escolar en Instituciones de Ecuador. Disponible en https://www.unicef.org/ecuador/acoso_escolar_final00 2.pdf

UNICEF. (2010). Clima, conflictos y violencia en la escuela.pdf. http://dide.minedu.gob.pe/bitstream/ handle/123456789/4547/Clima\%2C\%20conflictos\%20 y\%20violencia\%20en\%201a\%20escuela.pdf?sequence $=1 \&$ isAllowed $=\mathrm{y}$ 